\title{
Diastolic Augmentation Index Improves Augmentation Index in Assessing Arterial Stiffness
}

\author{
Yang $\mathrm{Yao}^{1}$, Lisheng $\mathrm{Xu}^{1 *}, \mathrm{Yu} \mathrm{Wang}^{1}$, Yahui Zhang ${ }^{1}$, Yingxian $\mathrm{Sun}^{2}$, Liang Guo ${ }^{2}$ \\ ${ }^{1}$ Sino-Dutch Biomedical and Information Engineering School, Northeastern University, \\ Shenyang City, Liaoning Province, 110819 China \\ 2 Department of cardiology, the First Hospital of China Medical University, Shenyang City, Liaoning \\ Province, 110001 China, China
}

\begin{abstract}
Arterial stiffness is an important risk factor for cardiovascular $(\mathrm{CV})$ events and is increasingly used in clinical practice. Radial augmentation index (AI) is used in assessing arterial stiffness, but is not only dependent on pulse wave velocity $(P W V)$, but also on several other factors like the reflect distance of pulse wave and height. This paper improved radial AI in assessing arterial stiffness by $d$-value, the subtraction between radial AI and diastolic AI (dAI). Nineteen subjects aged 18 to 80 years (mean $\pm S D, 46 \pm 27$ years) were enrolled in this study. Carotid-femoral PWV (cf-PWV), radial AI and dAI of each subject were measured. The $d$-value $(r=0.69$, $P<0.005)$ shows better linearity with cf-PWV than $A I$ $(r=0.62, \quad P<0.01)$ and $d A I$ (not significant) do. In conclusion, dAI improves AI in assessing arterial stiffness.
\end{abstract}

\section{Introduction}

Arterial stiffness is an important risk factor for cardiovascular (CV) events and is increasingly used clinically [1-4]. Many indicators have been proposed for the assessment of arterial stiffness. These indicators are basically classified into three types: systemic, regional and local arterial stiffness. In addition, wave reflection is also of great interest to estimate arterial stiffness.

Systemic arterial stiffness is available through use of a modified Windkessel model [5, 6], by use of the 'area method' $[7,8]$ or is simplified as the ratio between stroke volume (SV) and pulse pressure. However, these methods all involve an electrical model which is considered an inappropriate approximation of the circulation in the assessment of arterial stiffness [9]. In addition, the calculation of SV based on a single pulse wave (especially one at a peripheral site) is not well validated.

Local arterial stiffness is directly available (calculated as the change in volume divided by the change in pressure) at a specific site of the artery. The local arterial stiffness of superficial arteries is usually measured using echo-tracking devices. The carotid stiffness wins particular interest as is close to the aorta and is a site where atherosclerosis often occurs. However, its application in clinical is limited by the need of technical expertise. In addition, the carotid stiffness cannot be used as a surrogate of the aortic stiffness in patients with high blood pressure and/or diabetes [10]. The local arterial stiffness of deep arteries such as the ascending aorta is available through use of cine magnetic resonance imaging. However, the precision in determining the change of arterial diameter should be improved [11].

Regional arterial stiffness is usually assessed by the pulse wave velocity (PWV) of a specific segment of artery. Carotid-femoral PWV (cf-PWV) is considered as the 'gold standard' determinant of arterial stiffness $[1,11]$. However, limitations still exist. First, it is not convenient to record the carotid and femoral pulse waves simultaneously and patients should always keep in supine position. Second, the distance from the carotid to the femoral artery is difficult to measure accurately especially in patients with abdominal obesity [12]. In addition, the femoral pulse wave is not accurately available in patients with obesity, diabetes, metabolic syndrome, and/or peripheral artery disease [11].

Wave reflection is always used to estimate arterial stiffness. A pulse wave is composed of a forward and its corresponding reflected wave. The forward wave is generated by the pump of the heart, travels along the artery and is reflected at branch points or sites of impedance mismatch. The reflected wave then travels back and meets the forward wave. The reflected wave is added to the forward wave and cause augmentation in pulse pressure. This augmentation varies with PWV, which determines the time when the reflected wave meets the forward wave. When the artery is less stiff, the pulse wave velocity is low, the reflected wave meets the forward wave during diastole, whereas when the artery is stiffer, the reflected wave meets the forward wave during systole, highly augmented the pulse pressure. This 
augmentation caused by wave reflection is quantified by augmentation index (AI). Aortic AI has been shown to be an independent predictor of all-cause and CV mortality in end-stage renal failure patients [13]. Aortic AI corrected for heart rate (HR) of 75 bpm (AIx@ 75) has been proved to be independently associated with severe short- and long-term cardiovascular events in patients undergoing PCI [14]. However, aortic AI can hardly be obtained noninvasively. Radial augmentation index is conveniently available and is used to assess arterial stiffness in a broadly accepted device: HEM9000AI (Omron Healthcare, Japan). Radial augmentation index is shown to be predictive of $\mathrm{CV}$ events. Radial augmentation index is age-dependent and could be a useful index of vascular aging [15]. It is also reported that radial augmentation index is a predictor of premature coronary artery disease in younger males [16]. However, radial AI is not only dependent on PWV, but also on several other factors like the reflect distance of the pulse wave and height. In addition, it is shown that radial AI failed to measure vascular stiffness in the elderly over the age of 55 [17].

This paper improved radial AI in assessing arterial stiffness by the subtraction of radial AI and dAI. The subsequent contents are organized as follows: the second section describes the methodologies used in this study; the third section illustrates the results; the discussion and conclusion are demonstrated in the fourth section.

\section{Methodology}

\subsection{Study protocol}

Nineteem subjects aged 18 to 80 years (mean \pm SD, $46 \pm 27$ years) were enrolled in this study. Detailed information and physiologic conditions of the subjects are shown in Table 1. Subjects have a 10 min rest before the acquisition. During the acquisition procedure, subjects were kept in supine position all the time.

Carotid and femoral pulse waves were measured simultaneously using pressure pulse sensors. The data were recorded at a sampling frequency of $1000 \mathrm{~Hz}$. The PWV distance was measured using a tape from the carotid to the femoral artery. The radial pulse wave was recorded using the SphygmoCor device (AtCor, Australia) with a sampling frequency of $128 \mathrm{~Hz}$. AI and dAI were calculated from the radial pulse wave. To improve the accuracy, measurements of cf-PWV, AI and dAI were all repeated three times in each subject. Then the mean of the three measurements were taken to determine cf-PWV, AI and dAI of each subject.

\subsection{Data processing}

The carotid and femoral pulse waves should be preprocessed to eliminate baseline drift and noise, which
Table 1. Information of the subjects.

\begin{tabular}{ccccccc}
\hline No. & $\begin{array}{l}\text { Sex } \\
\text { (year) }\end{array}$ & Age & $\begin{array}{c}\text { Height } \\
(\mathrm{cm})\end{array}$ & $\begin{array}{c}\text { Weight } \\
(\mathrm{kg})\end{array}$ & $\begin{array}{c}\text { BP } \\
(\mathrm{mmHg})\end{array}$ & $\begin{array}{c}\text { HR } \\
(\mathrm{bpm})\end{array}$ \\
\hline 1 & $\mathrm{~F}$ & 23 & 161 & 48 & $102 / 68$ & 66 \\
2 & $\mathrm{~F}$ & 26 & 157 & 44 & $90 / 62$ & 64 \\
3 & $\mathrm{~F}$ & 19 & 172 & 61 & $118 / 70$ & 66 \\
4 & $\mathrm{~F}$ & 18 & 167 & 62 & $125 / 75$ & 74 \\
5 & $\mathrm{M}$ & 19 & 175 & 69 & $116 / 70$ & 60 \\
6 & $\mathrm{M}$ & 19 & 177 & 63 & $115 / 75$ & 66 \\
7 & $\mathrm{M}$ & 18 & 177 & 67 & $120 / 80$ & 74 \\
8 & $\mathrm{M}$ & 19 & 181 & 64 & $135 / 75$ & 73 \\
9 & $\mathrm{M}$ & 79 & 163 & 65 & $140 / 78$ & 80 \\
10 & $\mathrm{~F}$ & 60 & 160 & 60 & $120 / 64$ & 59 \\
11 & $\mathrm{~F}$ & 65 & 160 & 47 & $112 / 72$ & 80 \\
12 & $\mathrm{M}$ & 80 & 175 & 75 & $138 / 82$ & 66 \\
13 & $\mathrm{M}$ & 64 & 174 & 64 & $126 / 78$ & 86 \\
14 & $\mathrm{M}$ & 71 & 170 & 67 & $146 / 70$ & 53 \\
15 & $\mathrm{M}$ & 76 & 165 & 53 & $106 / 54$ & 61 \\
16 & $\mathrm{~F}$ & 80 & 160 & 63 & $134 / 60$ & 54 \\
\hline
\end{tabular}

highly influences the accuracy of subsequent calculation. Baseline drift is mainly brought in by body motion artifact and respiration. Wavelet decomposition was employed in drift removal. Wavelet decomposition at level 10 was applied to the data and the approximation coefficients were eliminated. Similarly, the noise was removed by applying wavelet decomposition at level 4 to the data and eliminating the detail coefficients.

\subsection{Calculation of PWV}

Carotid-femoral PWV was calculated by dividing travelled distance by pulse transit time (PTT). The distance was calculated as 0.8 times the direct distance from the right common carotid artery to the common femoral artery.

The PTT were calculated as the difference between the feet of the carotid and femoral pulse waves in time. The feet of the carotid and femoral pulse waves were both extracted using the intersecting tangents technique [1820], which determines the foot by the intersection of the horizontal line through the minimum and the tangent line through the maximum first derivative with respect to time.

PTT was obtained from each cardiac cycle in a series of data. In order to improve the accuracy and robustness of PTT calculation, the one exceeded the $90 \%$ of the SD distribution curve of the PTTs was discarded. The remaining PTTs were then averaged.

\subsection{Calculation of $\mathrm{AI}$ and $\mathrm{dAI}$}

As shown in Figure 1, AI is defined as the ratio between the second (P2) and first (P1) peak of radial 
pulse wave, and dAI is defined as diastolic peak (Pd) divided by P1. The difference between AI and dAI was named d-value, which means: d-value=AI-dAI.

AI was calculated automatically by the SphygmoCor device. dAI was calculated in each cardiac cycle. In each series of dAI derived from all cardiac cycles, the one exceeded the $90 \%$ of the SD distribution curve of dAI was discarded. The dAIs were then averaged to reduce the influence of respiration on the calculation of dAIs.

For each individual, the average radial pulse wave was derived using ensemble average method. Then, AI and dAI were both calculated from the averaged pulse wave.

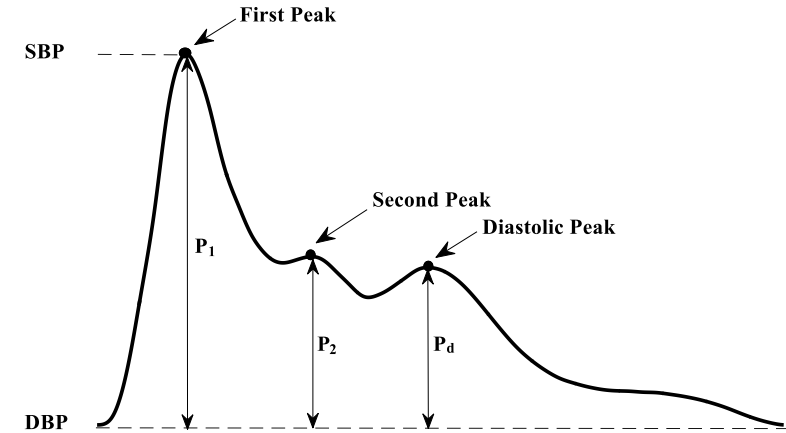

Figure 1. High-fidelity recording of the radial pulse wave. Amplitude of the peak and foot are the systolic (SBP) and diastolic (DBP) blood pressures, respectively. $\mathrm{P}_{1}$ indicates the difference between the first peak and the foot in amplitude; $\mathrm{P}_{2}$ is the amplitude of the second peak minus DBP; $P_{d}$ is the amplitude of the diastolic peak minus DBP.

\subsection{Statistical analysis}

To study the improvement of dAI on AI in assessing arterial stiffness, the linearity between d-value and cfPWV was compared with the ones between AI and cfPWV and between dAI and cf-PWV, respectively. The linearity was evaluated by correlation coefficients.

\section{Results}

\subsection{Reliability test}

To test the reliability of all the measurements, correlation coefficient between the first and second measurements of each parameter (cf-PWV, AI or dAI) in all subjects was calculated. The correlation coefficient between the first and second measurements of cf-PWV was $\mathrm{r}=0.97, \mathrm{P}<0.001$. The correlation coefficient between the first and second measurements of AI were $r=0.97$, $\mathrm{P}<0.001$ and $\mathrm{r}=0.87, \mathrm{P}<0.001$, respectively.

\subsection{Linearity between d-value and PWV}

Figure 2 illustrates the linearity between cf-PWV and
$\mathrm{AI}, \mathrm{dAI}$ and d-value, respectively. $\mathrm{AI}$ is significantly correlated with cf-PWV ( $\mathrm{r}=0.62, \mathrm{P}<0.005)$. dAI and cfPWV shows no significant linearity. Compared with AI, d-value significantly correlates with cf-PWV with a greater correlation coefficient $(r=0.68, \mathrm{P}<0.001)$.

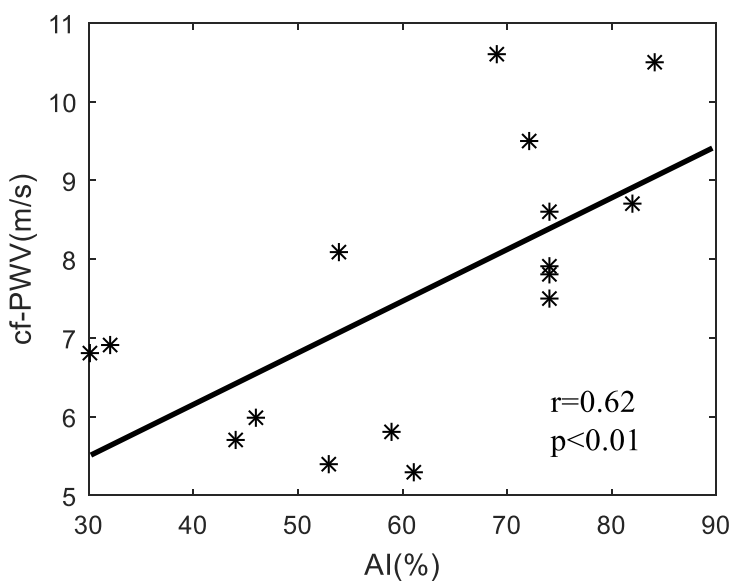

(a)

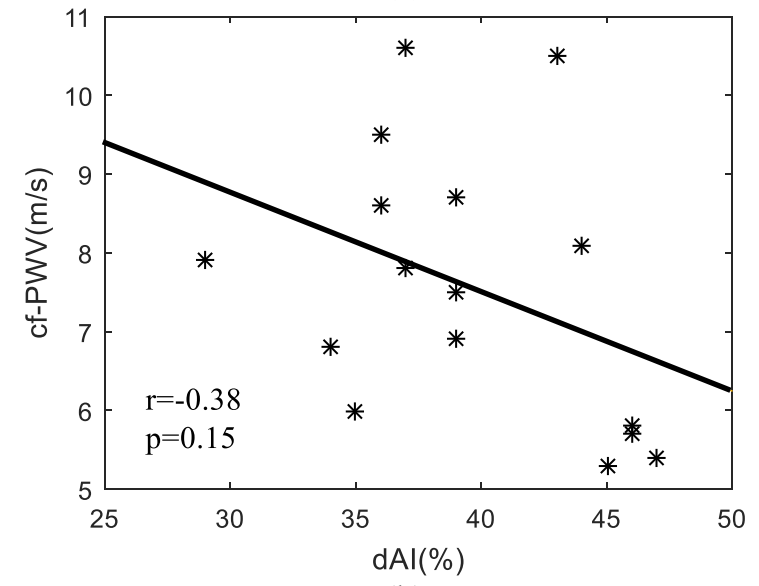

(b)

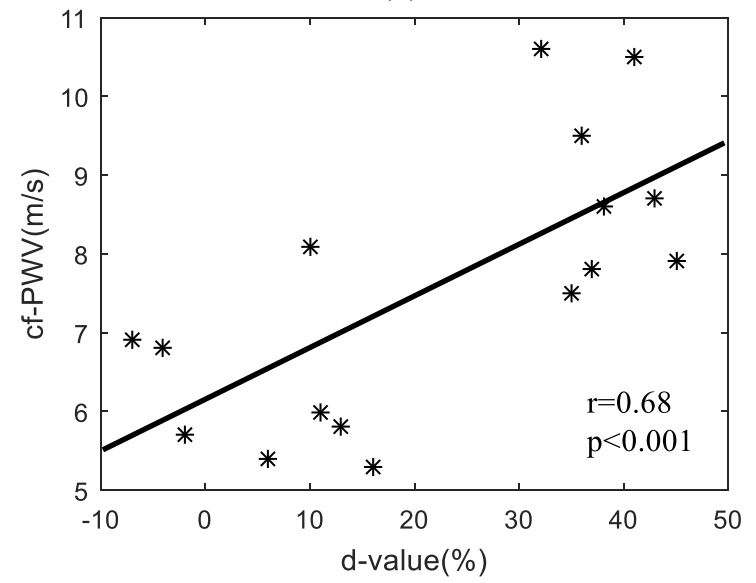

(c)

Figure 2. Performance comparison between AI, dAI and $\mathrm{d}$-value in assessing arterial stiffness: (a) linearity between AI and cf-PWV; (b) linearity between dAI and cf-PWV; (c) linearity between d-value and cf-PWV. 


\section{Discussion and conclusion}

This paper compared the performance of AI and dvalue in assessing arterial stiffness by studying their linearity with cf-PWV. AI shows positive correlation $(\mathrm{r}=0.62, \mathrm{P}<0.005)$ with cf-PWV whereas dAI shows no significant correlation with cf-PWV. d-value shows better linearity $(\mathrm{r}=0.68, \mathrm{P}<0.001)$ with cf-PWV than AI does. In conclusion, dAI indeed improved AI in assessing arterial stiffness. Further study is needed on the relationship between $\mathrm{d}$-value and $\mathrm{CV}$ events.

\section{Acknowledgements}

This work was supported by the National Natural Science Foundation of China (Nos. 61374015 and 61202258), and the Fundamental Research Funds for the Central Universities (Nos. N130404016 and N110219001).

\section{References}

[1] Mancia G, Fagard R, Narkiewicz K, Redon J, Zanchetti A, Böhm M, Christiaens T, Cifkova R, De Backer G, Dominiczak A, Galderisi M, Grobbee DE, Jaarsma T, Kirchhof P, Kjeldsen SE, Laurent S, Manolis AJ, Nilsson PM, Ruilope LM, Schmieder RE, Sirnes PA, Sleight P, Viigimaa M, Waeber B, Zannad F. 2013 ESH/ESC practice guidelines for the management of arterial hypertension. Blood Press 2014;23: 3-16.

[2] O'Rourke MF, O'Brien C, Edelman ER. Arterial Stiffening in Perspective: Advances in physical and physiological science over centuries. Am J Hypertens 2016; 20.

[3] Wakabayashi I. Homocysteine levels and arterial stiffness in the general population. J Atheroscler Thromb 2016; 11.

[4] García-Espinosa V, Curcio S, Castro JM, Arana M, Giachetto G, Chiesa P, Zócalo Y, Bia D. Children and adolescent obesity associates with pressure-dependent and age-related increase in carotid and femoral arteries' stiffness and not in brachial artery, indicative of nonintrinsic arterial wall alteration. Int J Vasc Med 2016;2016: 1-11.

[5] Cohn JN, Finkelstein S, McVeigh G, Morgan D, LeMay L, Robinson J, Mock J. Noninvasive pulse wave analysis for the early detection of vascular disease. Hypertension 1995;26: 503-8.

[6] McVeigh GE. Pulse waveform analysis and arterial wall properties. Hypertension 2003;41: 1010-1.

[7] Dart AM, Gatzka CD, Kingwell BA, Willson K, Cameron JD, Liang YL, Berry KL, Wing LM, Reid CM, Ryan P, Beilin LJ, Jennings GL, Johnston CI, McNeil JJ, Macdonald GJ, Morgan TO, West MJ. Brachial blood pressure but not carotid arterial waveforms predict cardiovascular events in elderly female hypertensives. Hypertension 2006;47: 785-90.

[8] Liu Z, Brin KP, Yin FC. Estimation of total arterial compliance: an improved method and evaluation of current methods. Am J Physiol 1986;251:H588-600.

[9] Pannier BM, Avolio AP, Hoeks A, Mancia G, Takazawa K. Methods and devices for measuring arterial compliance in humans. Am J Hypertens 2002;15: 743-53.

[10] Paini A, Boutouyrie P, Calvet D, Tropeano AI, Laloux B, Laurent S. Carotid and aortic stiffness: determinants of discrepancies. Hypertension 2006;47: 371-6.

[11] Laurent S, Cockcroft J, Van Bortel L, Boutouyrie P, Giannattasio C, Hayoz D, Pannier B, Vlachopoulos C, Wilkinson I, Struijker-Boudier H. Expert consensus document on arterial stiffness: methodological issues and clinical applications. Eur Heart J 2006;27: 2588-605.

[12] Van Bortel LM, Duprez D, Starmans-Kool MJ, Safar ME, Giannattasio C, Cockcroft J, Kaiser DR, Thuillez C. Clinical applications of arterial stiffness, Task Force III: recommendations for user procedures. Am J Hypertens 2002;15: 445-52.

[13] London GM1, Blacher J, Pannier B, Guérin AP, Marchais SJ, Safar ME. Arterial wave reflections and survival in endstage renal failure. Hypertension 2001;38: 434-8.

[14] Weber T, Auer J, O'rourke MF, Kvas E, Lassnig E, Lamm G, Stark N, Rammer M, Eber B. Increased arterial wave reflections predict severe cardiovascular events in patients undergoing percutaneous coronary interventions. Eur Heart J 2005;26: 2657-63.

[15] Kohara K, Tabara Y, Oshiumi A, Miyawaki Y, Kobayashi T, Miki T. Radial augmentation index: a useful and easily obtainable parameter for vascular aging. Am J Hypertens 2005;18: 11S-14S

[16] Fischer-Rasokat U, Brenck F, Zeiher AM, Spyridopoulos I. Radial augmentation index unmasks premature coronary artery disease in younger males. Blood Press Monit 2009;14: 59-67.

[17] Fantin F, Mattocks A, Bulpitt CJ, Banya W, Rajkumar C, Is augmentation index a good measure of vascular stiffness in the elderly?, Age Ageing 2007;36:43-8.

[18] Mukkamala R, Hahn JO, Inan OT, Mestha LK, Kim CS, Töreyin H, Kyal S. Toward Ubiquitous Blood Pressure Monitoring via Pulse Transit Time: Theory and Practice. IEEE Trans Biomed Eng. 2015;62: 1879-901.

[19] Gaddum NR1, Alastruey J, Beerbaum P, Chowienczyk P, Schaeffter T. A technical assessment of pulse wave velocity algorithms applied to non-invasive arterial waveforms. Ann Biomed Eng. 2013;41: 2617-29.

[20] Zhang G, Gao M, Xu D, Olivier NB, Mukkamala R. Pulse arrival time is not an adequate surrogate for pulse transit time as a marker of blood pressure. J Appl Physiol 2011;111: 1681-6.

Address for correspondence.

Prof. Lisheng Xu.

Sino-Dutch Biomedical and Information Engineering School, Northeastern University, Shenyang City, Liaoning Province, 110819 China.

E-mail: xuls@bmie.neu.edu.cn. 